\title{
The nasal cavity microbiota of healthy adults
}

\author{
Christine M Bassis ${ }^{1 *}$, Alice L Tang ${ }^{2}$, Vincent B Young ${ }^{1}$ and Melissa A Pynnonen ${ }^{3}$
}

\begin{abstract}
Background: The microbiota of the nares has been widely studied. However, relatively few studies have investigated the microbiota of the nasal cavity posterior to the nares. This distinct environment has the potential to contain a distinct microbiota and play an important role in health.

Results: We obtained 35,142 high-quality bacterial 16S rRNA-encoding gene sequence reads from the nasal cavity and oral cavity (the dorsum of the tongue and the buccal mucosa) of 12 healthy adult humans and deposited these data in the Sequence Read Archive (SRA) of the National Center for Biotechnology Information (NCBI) (Bioproject: PRJNA248297). In our initial analysis, we compared the bacterial communities of the nasal cavity and the oral cavity from ten of these subjects. The nasal cavity bacterial communities were dominated by Actinobacteria, Firmicutes, and Proteobacteria and were statistically distinct from those on the tongue and buccal mucosa. For example, the same Staphylococcaceae operational taxonomic unit (OTU) was present in all of the nasal cavity samples, comprising up to $55 \%$ of the community, but Staphylococcaceae was comparatively uncommon in the oral cavity.

Conclusions: There are clear differences between nasal cavity microbiota and oral cavity microbiota in healthy adults. This study expands our knowledge of the nasal cavity microbiota and the relationship between the microbiota of the nasal and oral cavities.
\end{abstract}

Keywords: Nasal cavity, Oral cavity, Tongue, Buccal mucosa, Microbiota, Culture-independent

\section{Background}

Bacterial communities play important roles in the health of their hosts, including roles in immune system development [1], nutrition [2], and resistance to infection [3]. In this study, we compared the nasal cavity microbiota and the oral cavity microbiota from healthy adult humans. Until recently, the bacterial community of the healthy human nasal cavity had not been characterized by culture-independent methods $[4,5]$. However, many studies have characterized the microbiota of the healthy human nares [6-11], which are adjacent and anterior to the nasal cavity. Staphylococcus aureus carriage in the nares is linked to increased risk of $S$. aureus infection in other body sites $[12,13]$. Further, antagonism by and competition with other members of the nares microbiota seem to influence $S$. aureus nares carriage [12]. Although adjacent to the nares, the nasal cavity is distinct from the nares with a different type of epithelium, a

\footnotetext{
*Correspondence: cbassis@umich.edu

'Department of Internal Medicine, Division of Infectious Diseases, University of Michigan, 1510 MSRB I SPC 5666, 1150 W. Medical Center Dr, Ann Arbor, Ml 48109-5666, USA

Full list of author information is available at the end of the article
}

non-keratinized stratified squamous epithelium that transitions to a typical respiratory epithelium-ciliated pseudostratified columnar epithelial cells and mucusproducing goblet cells [14]. In contrast, the nares have a keratinized, stratified squamous epithelium with hairs and sebaceous glands. Relatively few studies have investigated the bacterial community composition of the nasal cavity in healthy humans. In this study, we sought to expand our knowledge of the healthy human nasal cavity microbiota and compare the nasal cavity microbiota to the oral cavity microbiota in the same subjects.

\section{Methods}

\section{Subject recruitment and characteristics}

This study was approved by the University of Michigan Institutional Review Board. All subjects provided written informed consent. Twelve adults patients were recruited from a tertiary care otolaryngology clinic (Additional file 1: Table S1). Exclusion criteria were patients who had acute or chronic sinusitis and patients who were taking antibiotics or oral steroids for any reason. 


\section{Sampling}

4N6 DNA flocked swabs (Cat. No. 3520CA, Copan Diagnostics Inc., Murrieta, CA, USA) were used to sample all sites. The nasal cavity was sampled by inserting the swab into the nasal passage between the septum and middle turbinate, taking care to avoid contact to the nares. The dorsum of the tongue and buccal mucosa were sampled with separate swabs. The samples were transferred directly into the Eppendorf tubes provided with the swab and stored on ice and then at $-20^{\circ} \mathrm{C}$ until DNA isolation.

\section{DNA isolation}

DNA was isolated from the swabs with a PowerSoil DNA isolation kit (Mo Bio Laboratories, Inc., Carlsbad, CA, USA) according to the manufacturer's instructions except that 2 min of bead beating using the 'Homogenize' setting of a Mini-BeadBeater-8 (Biospec Products, Bartlesville, OK, USA) was done in place of $10 \mathrm{~min}$ of vortexing.

\section{Primary PCR amplification, pooling, and sequencing}

We based our protocol for amplifying and preparing libraries of the V5V3 region of the $16 \mathrm{~S}$ rRNA-encoding gene on HMP 16S Protocol Version 4.2 (http://www. hmpdacc.org/doc/16S_Sequencing_SOP_4.2.2.pdf). Each $20 \mu \mathrm{l}$ polymerase chain reaction (PCR) reaction contained $2 \mu \mathrm{l}$ AccuPrime PCR Buffer II (Invitrogen, Carlsbad, CA, USA), $0.15 \mu \mathrm{l}$ AccuPrime Taq DNA Polymerase High Fidelity (Invitrogen), $0.2 \mu \mathrm{M}$ primer A (CCATCTCATCC CTGCGTGTCTCCGACTCAGXXXXXCCGTCAATTCM TTTRAGT), $0.2 \mu \mathrm{M}$ primer B (CCTATCCCCTGTGTG CCTTGGCAGTCTCAGCCTACGGGAGGCAGCAG), and $1 \mu \mathrm{l}$ DNA for the oral cavity samples or $15.45 \mu \mathrm{l}$ DNA for the nasal samples. The bold portions of primer A and primer B are 926R and $357 \mathrm{~F}$, respectively. The region of primer A represented by XXXXX is the 5-10 nucleotide barcode sequence. The remainder of primer $\mathrm{A}$ and primer $B$ are the $A$ adapter sequence and the $B$ adapter sequence, respectively, required for emPCR and 454 sequencing. The PCR was run for $2 \mathrm{~min}$ at $95^{\circ} \mathrm{C}$ followed by 30 cycles of $95^{\circ} \mathrm{C}$ for $20 \mathrm{~s}, 50^{\circ} \mathrm{C}$ for $30 \mathrm{~s}$, and $72^{\circ} \mathrm{C}$ for $5 \mathrm{~min}$. The PCR products were purified with AMPure XP (Agencourt Bioscience Corporation, Beckman Coulter, Inc., Beverly, MA, USA) according to the manufacturer's instructions except $0.6 \times$ the amplicon volume $(10.8 \mu \mathrm{l})$ of beads was used rather than $1.2 \times$ in order to remove more of the small products. The purified PCR products were quantified with a Quant-iT PicoGreen dsDNA kit (Invitrogen) according to the manufacturer's instructions and combined into a pool with equal amounts of each amplicon. The pool was then purified with AMPure XP (Agencourt Bioscience Corporation) according to the manufacturer's instructions except the volume of beads was $0.6 \times$ the pool volume. The pool was quantified with a Library
Quantification Kit for Roche 454 GS Titanium (Kapa Biosystems, Inc., Wilmington, MA, USA). A Junior emPCR (454 Life Sciences, Roche, Branford, CT, USA) was performed, and 454 sequencing was done on a GS Junior (454 Life Sciences) according to manufacturer's instructions.

\section{Sequence processing}

Sequences were processed with mothur v.1.28.0 according to the Schloss SOP of November 27, $2012[15,16]$. In summary, the sff file was input to sffinfo, trim.flows was run allowing 1 mismatch in the barcode and 2 mismatches in the $926 \mathrm{R}$ region of the primer, and sequencing error was reduced with shhh.flows. With trim.seqs, barcode and primer sequences were removed and all sequences less than 200 bases or with homopolymers longer than eight nucleotides were discarded. The sequences were aligned to the Silva reference alignment $[17,18]$. In order to compare sequences over the same region of the alignment, we set the end position at 27659 and chose a start position that was met by $95 \%$ of the sequences. With pre.cluster, sequences within two base pairs were merged. Chimeras were identified with chimera.uchime [19] and removed. The sequences were classified using a modified form of RDP training set version 9 (trainset9_032012.pds.tax and trainset9_032012.pds.fasta) [20]. Sequences classified as Chloroplast, Mitochondria, Archaea, Eukaryota, or unknown kingdom were removed.

\section{Sequence analysis}

For our initial analysis, we included subjects only if sequences were obtained for a complete set of samples (buccal mucosa, tongue, and nasal cavity). Therefore, in order to maximize the number of subjects included in the study, we decided to subsample 269 sequences from each sample. So, subjects were included in our initial analysis only if at least 269 sequences were obtained from all three sites (nasal cavity, buccal mucosa, and dorsal side of tongue). A distance matrix made with dist. seqs was used with the average neighbor algorithm to group sequences into operational taxonomic units (OTUs) with the cluster command. OTUs defined as 3\% different were used for further analysis. The make.shared command was used to produce a table (shared file) of the number of sequence reads assigned to each OTU in each sample. The shared file was used to calculate $\theta$ $(1-\theta$ similarity index), a metric of community dissimilarity that takes the relative abundances of both shared and non-shared OTUs into account [21]. Principle coordinates analysis (PCoA) was used to visualize the $\theta$ distance matrix, and analysis of molecular variance (AMOVA) [22] was used to test the statistical significance of the differences between bacterial communities 
of different groups (i.e., nasal cavity communities versus buccal mucosa communities).

\section{Quality assurance}

To ensure that the source of bacterial sequences was not the swab itself or the DNA isolation reagents, PCR was performed on DNA isolated from an unused swab. To confirm that the PCR reagents were not the source of bacterial sequences, PCR of the no-template control was performed. Neither of these control PCRs yielded products visible on a gel, indicating that there was minimal contamination from the swab or reagents.

\section{Initial findings}

\section{Sequences obtained}

After sequence processing, we obtained a total of 35,142 high-quality bacterial $16 \mathrm{~S}$ rRNA-encoding gene sequence reads from 35 buccal mucosa, tongue (dorsal side), and nasal cavity samples from 12 subjects with a mean of 1,115 sequences per sample (Additional file 1: Table S1). The mean sequence length after sequence processing was 268 bases. The number of sequences obtained per sample from the buccal mucosa microbiota ranged from 715-1,684. The number of sequences obtained per sample from the tongue microbiota ranged from 519-1,597. The number of sequences obtained per sample from the nasal cavity microbiota ranged from 1-
1,595. The sff files from which the processed sequences were generated were submitted to the SRA (Bioproject: PRJNA248297) except for the sff that yielded only one sequence (nasal cavity L) (Additional file 1: Table S1).

\section{Nasal cavity bacterial communities were distinct from the} bacterial communities on the tongue and buccal mucosa

For our initial analysis, we subsampled 269 sequences from each sample following sequence processing. Ten healthy adult subjects had at least 269 sequences from each sampling site following sequence processing (nasal cavity, dorsum of the tongue, and buccal mucosa) and were included in the analysis (Additional file 1: Table S1). As illustrated by PCoA of $\theta$ dissimilarities, the bacterial communities clustered based on body site, with the nasal cavity bacterial communities more similar to each other than to the oral cavity bacterial communities (Figure 1). Based on $\theta$ dissimilarities, the differences between the bacterial communities of the nasal cavity and each oral cavity subsite were statistically significant (AMOVA $p$ value $<0.001$ for each comparison). Additionally, although the bacterial communities of the two sites in the oral cavity were more similar to each other than to the nasal cavity communities, based on $\theta$ dissimilarities, the bacterial communities from the tongue and the buccal mucosa were distinct (AMOVA $p$ value $<0.001)$.

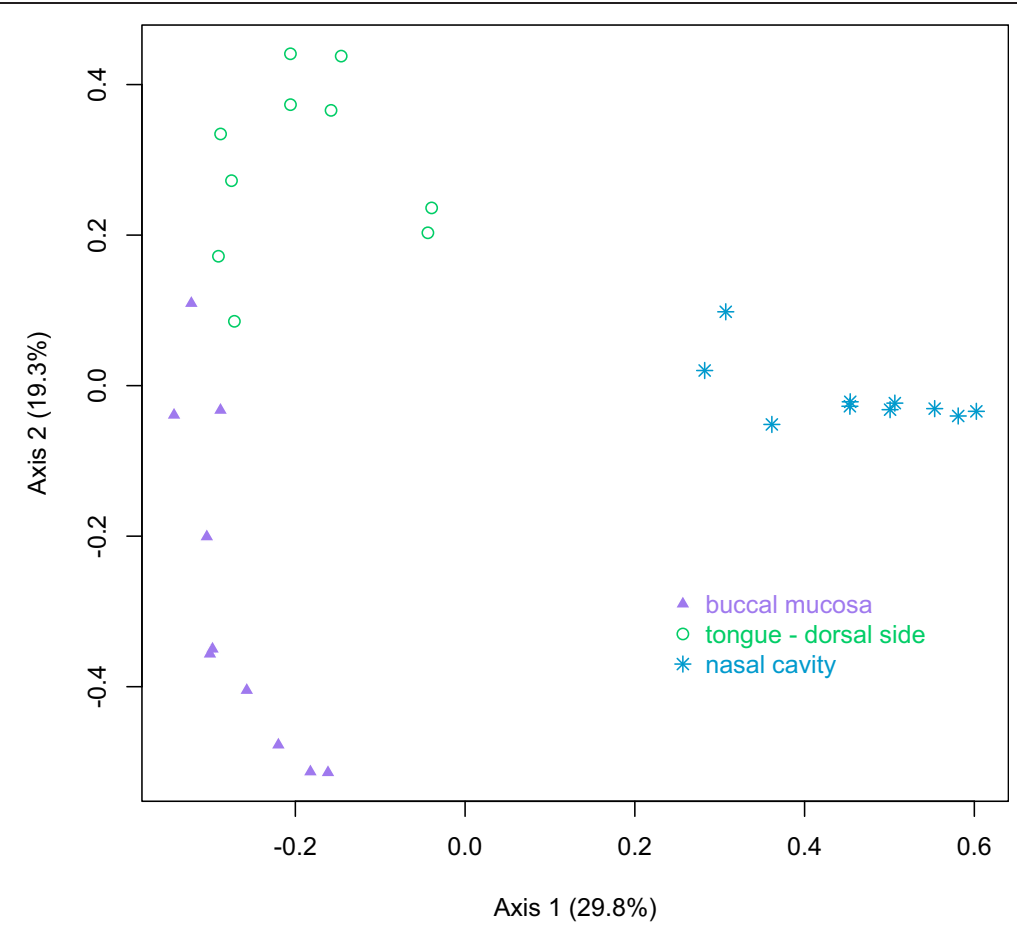

Figure 1 Principal coordinates analysis of nasal cavity, buccal mucosa and tongue microbiota. Principal coordinates analysis was performed on a matrix of $\theta$ distances between all samples. OTUs were defined as 3\% different. Axis 1, representing $29.8 \%$ of the variance, and axis 2, representing $19.3 \%$ of the variance, are displayed. 


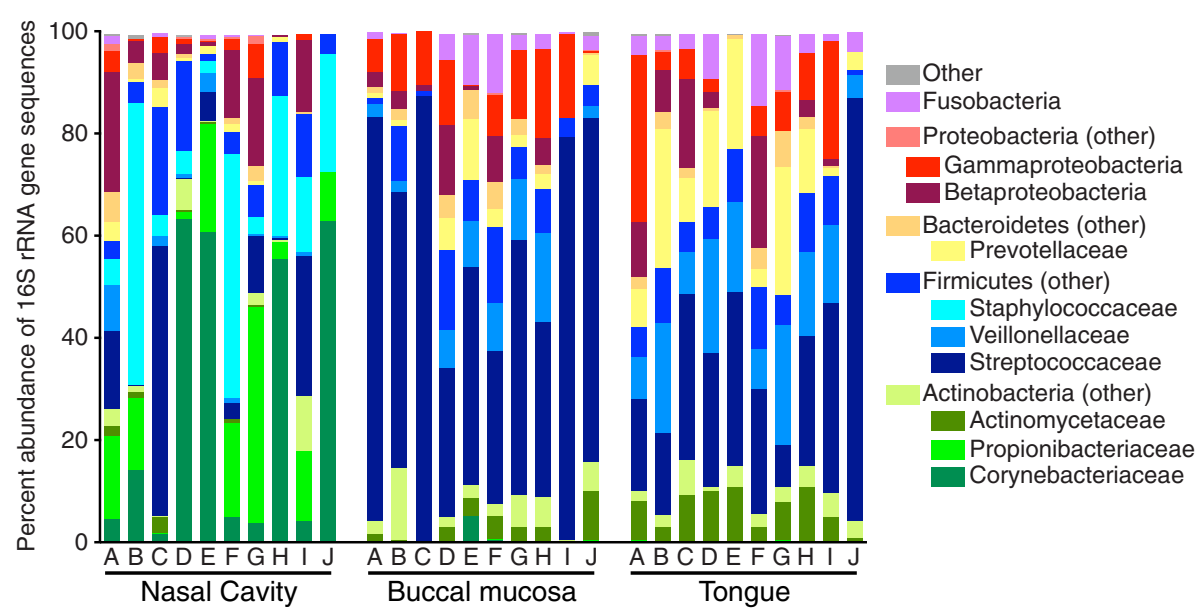

Figure 2 Bacterial community composition of the nasal cavity, buccal mucosa, and tongue. Two hundred sixty-nine sequences were subsampled from each sample. The sequences were then classified as described in the materials and methods, and the relative abundances of sequences classified to major taxonomic groups are displayed.

\section{Composition of the nasal cavity microbiota}

In our study and other recent studies [4,5], the bacterial communities of the nasal cavities were dominated by Actinobacteria, Firmicutes, and in some cases, Proteobacteria (Figure 2). Corynebacteriaceae and Propionibacteriaceae were the most prevalent families of Actinobacteria in the nasal cavity (Figure 2). Between subjects, the levels of Corynebacteriaceae varied from $1.5 \%$ to $62.8 \%$, and the levels of Propionibacteriaceae varied from $0.4 \%$ to $42.4 \%$ (Figure 2, Additional file 2: Table S2). Actinobacteria were present at lower levels in the communities at both oral cavity sites, but they were from the families Micrococcaceae and Actinomycetaceae. Corynebacteriaceae composed over $1 \%$ of the community in only one oral cavity sample. Propionibacteriaceae was undetectable in most oral cavity samples, and when it was detected, it never exceeded $1 \%$ of the community.

Another striking difference between the nasal cavity and oral cavity communities was the composition of the Firmicutes. The same Staphylococcaceae OTU was present in the nasal cavity samples of all subjects, ranging from $2.2 \%$ $55.0 \%$ of the community, while Staphylococcaceae were not detected in the oral cavity communities (Additional file 2: Table S2). It was not possible to distinguish Staphylococcus aureus and Staphylococcus epidermidis in the region of the $16 \mathrm{~S}$ rRNA gene covered by our sequences. Generally, in the oral cavity, Streptococcaceae was the most abundant Firmicutes family, and in several subjects, the most abundant family overall. Veillonellaceae was also more abundant in the oral cavity.

The overall levels of Betaproteobacteria were not significantly different between nasal cavity communities and oral cavity communities (Kruskal-Wallis test, $p$ value $=0.4557$ ). However, the composition of the Betaproteobacteria differed between the nasal cavity and the oral cavity: all three sites included Neisseriaceae at levels that were not statistically different (Kruskal-Wallis test, $p$ value $=0.4543$ ), but the nasal cavity contained higher levels of Comamonadaceae (Kruskal-Wallis test, $p$ value $=0.0002$ ) and Burkholderiales incertae sedis (Kruskal-Wallis test, $p$ value $=$ $0.0004)$.

\section{Future directions}

With its proximity to the sinuses-the maxillary sinuses communicate with the nasal cavity through 1 to $2-\mathrm{mm}$ ostia-the nasal cavity microbiota might be a useful proxy for the less accessible sinus microbiota. Signatures of sinusitis in the sinus microbiota have recently been identified [23]. To determine if specific changes in the nasal cavity microbiota also accompany sinusitis and could be used as an indicator of sinus infections, it will be necessary to sample both the nasal cavity and the sinus in the same subjects with and without sinusitis.

\section{Availability of supporting data}

The sff files have been deposited in the SRA (Bioproject: PRJNA248297).

\section{Additional files}

Additional file 1: Table S1. Number of sequences obtained from each sample and subject characteristics.

Additional file 2: Table S2. Relative abundances of bacterial families in each sample based on classification of 165 rRNA-encoding gene sequence reads.

\section{Abbreviations}

OTU: Operational taxonomic unit; PCoA: Principal coordinates analysis; PCR: Polymerase chain reaction.

\section{Competing interests}

The authors declare that they have no competing interests. 


\section{Authors' contributions}

The study was designed by ALT, VBY, and MAP. ALT and MAP did the sample collection. DNA isolation was done by ALT. Sequencing library preparation and sequence analysis were performed by CMB. CMB and MAP wrote the manuscript. CMB, ALT, VBY, and MAP edited the manuscript. All authors read and approved the final manuscript.

\section{Acknowledgements}

This study was supported, in part, by funding from the National Heart, Lung, and Blood Institute of the NIH (5U01HL098961 and 5T32HL07749). We would like to thank Susan Foltin for DNA sequencing and Lauren Miller for PCR troubleshooting.

\section{Author details}

${ }^{1}$ Department of Internal Medicine, Division of Infectious Diseases, University of Michigan, 1510 MSRB I SPC 5666, 1150 W. Medical Center Dr, Ann Arbor, Ml 48109-5666, USA. ²Department of Otolaryngology, University of Cincinnati, Medical Sciences Building, 231 Albert Sabin Way, Cincinnati, OH 45267-0528, USA. ${ }^{3}$ Department of Otolaryngology, University of Michigan, 1500 E. Medical Center Drive, Taubman Center 1904, Ann Arbor, MI 48109-0312, USA.

Received: 7 March 2014 Accepted: 26 June 2014

Published: 11 August 2014

\section{References}

1. Erturk-Hasdemir D, Kasper DL: Resident commensals shaping immunity. Curr Opin Immunol 2013, 25:450-455.

2. Flint HJ, Scott KP, Louis P, Duncan SH: The role of the gut microbiota in nutrition and health. Nat Rev Gastroenterol Hepatol 2012, 9:577-589.

3. Theriot CM, Young VB: Microbial and metabolic interactions between the gastrointestinal tract and Clostridium difficile infection. Gut Microbes 2014, 5:86-95.

4. Yan M, Pamp SJ, Fukuyama J, Hwang PH, Cho D-Y, Holmes S, Relman DA: Nasal microenvironments and interspecific interactions influence nasal microbiota complexity and S. aureus carriage. Cell Host Microbe 2013, 14:631-640.

5. Ramakrishnan VR, Feazel LM, Gitomer SA, Ir D, Robertson CE, Frank DN: The microbiome of the middle meatus in healthy adults. PLoS One 2013, 8:e85507.

6. The Human Microbiome Project Consortium: Structure, function and diversity of the healthy human microbiome. Nature 2012, 486:207-214

7. Costello EK, Lauber CL, Hamady M, Fierer N, Gordon Jl, Knight R: Bacterial community variation in human body habitats across space and time. Science 2009, 326:1694-1697.

8. Grice EA, Kong HH, Conlan S, Deming CB, Davis J, Young AC, Comparative Sequencing Program NISC, Bouffard GG, Blakesley RW, Murray PR, Green ED, Turner ML, Segre JA: Topographical and temporal diversity of the human skin microbiome. Science 2009, 324:1190-1192. 10.1126/science.1171700.

9. Lemon KP, Klepac-Ceraj V, Schiffer HK, Brodie EL, Lynch SV, Kolter R: Comparative analyses of the bacterial microbiota of the human nostril and oropharynx. mBio 2010, 1(3):e00129-10.

10. Frank DN, Feazel LM, Bessesen MT, Price CS, Janoff EN, Pace NR: The human nasal microbiota and Staphylococcus aureus carriage. PLoS One 2010, 5:e10598.

11. Oh J, Conlan S, Polley E, Segre J, Kong H: Shifts in human skin and nares microbiota of healthy children and adults. Genome Med 2012, 4:77.

12. Verhoeven PO, Gagnaire J, Botelho-Nevers E, Grattard F, Carricajo A, Lucht F, Pozzetto B, Berthelot P: Detection and clinical relevance of Staphylococcus aureus nasal carriage: an update. Expert Rev Anti Infect Ther 2014, 12:75-89.

13. Wertheim HFL, Vos MC, Ott A, van Belkum A, Voss A, Kluytmans JA, van Keulen PH, Vandenbroucke-Grauls CM, Meester MH, Verbrugh HA: Risk and outcome of nosocomial Staphylococcus aureus bacteraemia in nasal carriers versus non-carriers. Lancet 2004, 364:703-705.

14. Mescher AL, Mescher AL: The respiratory system. In Junqueira's Basic Histology: Text and Atlas. 13th edition. New York: McGraw-Hill; 2013. http://accessmedicine.mhmedical.com/content.aspx?bookid=574\&Sectionid= 42524603]. Accessed July 03, 2014.

15. Schloss PD, Westcott SL, Ryabin T, Hall JR, Hartmann M, Hollister EB, Lesniewski RA, Oakley BB, Parks DH, Robinson CJ, Sahl JW, Stres B, Thallinger GG, Van Horn DJ, Weber CF: Introducing mothur: open-source, platform-independent, community-supported software for describing and comparing microbial communities. Appl Environ Microbio/ 2009, 75:7537-7541.

16. Schloss PD, Gevers D, Westcott SL: Reducing the effects of PCR amplification and sequencing artifacts on 16S rRNA-based studies. PLoS One 2011, 6:e27310

17. Quast C, Pruesse E, Yilmaz P, Gerken J, Schweer T, Yarza P, Peplies J, Glöckner FO: The SILVA ribosomal RNA gene database project: improved data processing and web-based tools. Nucleic Acids Res 2013, 41:D590-D596.

18. Schloss PD: A high-throughput DNA sequence aligner for microbial ecology studies. PLoS One 2009, 4:e8230.

19. Edgar RC, Haas BJ, Clemente JC, Quince C, Knight R: UCHIME improves sensitivity and speed of chimera detection. Bioinformatics 2011, 27:2194-2200.

20. Cole JR, Chai B, Farris RJ, Wang Q, Kulam SA, McGarrell DM, Garrity GM, Tiedje JM: The Ribosomal Database Project (RDP-II): sequences and tools for high-throughput rRNA analysis. Nucleic Acids Res 2005, 33:D294-D296.

21. Yue JC, Clayton MK: A similarity measure based on species proportions. Commun Stat-Theory Methods 2005, 34:2123-2131.

22. Anderson MJ: A new method for non-parametric multivariate analysis of variance. Austral Ecol 2001, 26:32-46.

23. Abreu NA, Nagalingam NA, Song Y, Roediger FC, Pletcher SD, Goldberg AN, Lynch SV: Sinus microbiome diversity depletion and Corynebacterium tuberculostearicum enrichment mediates rhinosinusitis. Sci Trans/ Med 2012, 4:151ra124.

doi:10.1186/2049-2618-2-27

Cite this article as: Bassis et al:: The nasal cavity microbiota of healthy adults. Microbiome 2014 2:27.

\section{Submit your next manuscript to BioMed Central and take full advantage of:}

- Convenient online submission

- Thorough peer review

- No space constraints or color figure charges

- Immediate publication on acceptance

- Inclusion in PubMed, CAS, Scopus and Google Scholar

- Research which is freely available for redistribution

Submit your manuscript at www.biomedcentral.com/submit
C) Biomed Central 